\section{Svennik Høyer / Horst Pöttker (eds.) Diffusion of the News Paradigm 1850 / 2000}

Göteborg: Nordicom Göteborg University 2005. - $311 \mathrm{~S}$.

\section{ISBN 91-89471-30-X}

Dient Mediengeschichtsschreibung international als ein Laboratorium? Lässt sich die Bedeutung zentraler Traditionen, die den Journalismus beeinflussen, vergleichend analysieren? Die Herausgeber Svennik Høyer und Horst Pöttker jedenfalls postulieren ein NachrichtenParadigma und untersuchen es für die beiden letzten Jahrhunderte. Insgesamt achtzehn Artikel beleuchten historische, politische, kulturelle sowie auch ideologiekritische Aspekte.

Svennik Høyer postuliert mit Verweis auf die seinerzeit bahnbrechende Abhandlung von Thomas S. Kubn „The Structure of Scientific Revolutions" für das „Nachrichten-Paradigma" fünf Elemente: „The Event, News Value Factors, The News Interview, The Inverted Pyramid, Journalistic Objectivity“ (vgl. S. 11). Anschließend formuliert Høyer vier Ausgangshypothesen: 1. Das Nachrichten-Paradigma ist nicht universell. Vielmehr wurzelt es in historischen und sozialen Bedingungen: Jeweils unterschiedliche Ideen und kulturelle Werte werden toleriert. 2. In Konfliktsituationen und in Diktaturen leiden journalistische Objektivität und Autonomie. 3. Meinungs- und Redefreiheit sind essenziell für die Diffusion des Nachrichten-Paradigmas. 4. Diese folgt nicht einer linearen Ursache-Wirkungs-Beziehung. Sie setzt jeweils unter verschiedenen nationalen und kulturellen Hintergründen spezifisch ein.

Im anglo-amerikanischen Kontext analysiert Michael Schudson, wie die Objektivitätsnorm artikuliert und begründet wird. Sie repräsentiert auch organisatorische Bedingungen sozialer Kontrolle. Journalismus wird $\mathrm{zu}$ einer Berufskultur, welche Objektivität als professionelle Norm einzuhalten versucht.

Harlan S. Stensaas („The Rise of the News Paradigm") analysiert Merkmale des Nachrichten-Paradigmas. Wesentliche Determinanten sind Technologie, Public Relations, Kommerzialisierung sowie Professionalisierung. Für den kulturellen Kontext eines ,neuen Journalismus' sei der Aufschwung der wissenschaftlichen Methode ausschlaggebend: Objektivität ließe sich so vergrößern.

Horst Pöttker („The News Pyramid and its
Origins from the American Journalism in the $19^{\text {th }}$ Century") beschreibt Nachrichten als eine narrative Struktur: Überschrift und Intro beantworteten jeweils die klassischen W-Fragen. Außerdem wird das aktuellste Ereignis an vorderster Stelle platziert. Dieses Prinzip erklärt sich aus technologischen Bedingungen einer zunächst unzuverlässigen Telegraphenübermittlung sowie aus Motiven der politischen Steuerung über eine effektive Beeinflussung der Öffentlichkeit. Schließlich gibt es eine ökonomische Erklärung: Notwendig sind Kostenersparnis und Marktorientierung bei zunehmend knapper Aufmerksamkeit. Pöttker untersucht die Durchsetzung der „Inverted Pyramid" anhand einer Inhaltsanalyse von New York Herald und New York Times in den Jahren 1865 bis 1920. Dabei zeigt sich, dass sich der Anteil pyramidial verfasster Nachrichten versechsfacht. Svennik Høyer untersucht anschließend „Old and New Journalism in the London Press“ zu Ende des 19. Jahrhunderts. Der neue Journalismus in England wird durch die amerikanischen Vorläufer stark beeinflusst, aber nicht durch sie dominiert.

Nachfolgend präsentiert der Band für Skandinavien, Deutschland sowie Zentral- und Osteuropa elf Fallstudien. Sie beschäftigen sich - allerdings nicht einheitlich und systematisch - mit einzelnen Elementen des NachrichtenParadigmas. Søren Kolstrup arbeitet am Beispiel Dänemarks das Relevanzprinzip heraus: Es löst eine reine Erzählstruktur ab und wird offensichtlich erstmalig während des amerikanischen Bürgerkrieges praktiziert. Das Relevanzprinzip gestattet dem Leser, selektiv zu lesen. Außerdem können Herausgeber lange Artikel ohne Informationsverlust kürzen.

Horst Pöttker beschreibt für Deutschland die Tradition des politischen Parteienjournalismus, Journalismus wird als Politik verstanden. Für die Entstehung des Parteienjournalismus sind mit Verweis auf Untersuchungen von Wolfgang Donsbach zwei Gründe verantwortlich: die Aufklärung mit ihrer Neigung zu philosophischen, kulturellen und politischen Ermahnungen sowie die erst später in Deutschland realisierte Pressefreiheit.

Rudolf Stöber zeichnet differenziert Erfindung, Differenzierung und Verbreitung der Presse nach - beginnend mit Gutenberg. Stöber verwendet strukturelle Indikatoren, so zum Beispiel die Zahl der erschienenen deutschen Zeitungen (ab 1605) sowie die Preis- 
entwicklung der Zeitungen im 18. Jahrhundert. Zeitungen werden zunächst u. a. durch höchste Richter, reiche Kaufleute sowie Staatsoberhäupter organisiert - der Klerus sowie Führungseliten der frühen modernen Staaten dominieren. Journalismus unterscheidet sich von verwandten Berufen, insbesondere von literarischen Autoren. Die wollen allerdings nicht "Tagesschriftsteller“ sein. Stöber zeigt, wie sich das Pressegeschäft vom Buchgeschäft emanzipiert: Herausgeber geben dabei bestimmte redaktionelle Prinzipien vor. Im internationalen Vergleich ist für Deutschland die Berufsgeschichte des Journalismus vergleichsweise gut dokumentiert; Stöber kann auf exzellente regionale Studien, in Thüringen etwa auf Werner Greiling mit der im Jahr 2003 erschienenen Abhandlung „Presse und Öffentlichkeit in Thüringen - Mediale Verdichtung und kommunikative Vernetzung im 18. und 19. Jahrhundert" zurückgreifen.

Bernd Blöbaum charakterisiert die Entwicklung des westdeutschen Journalismus nach 1945 als System: Es konstituiert sich aus bestimmten organisatorischen Eigenheiten, speziellen Rollen sowie bestimmten Produktionsregeln. Durch die Einflüsse der Alliierten konnte sich in Deutschland ein modernes Nachrichten-Paradigma entwickeln; es reflektiert britische und amerikanische Konzepte. Exklusivität und Aktualität werden zunehmend essenzielle Kriterien im Wettbewerb der Massenmedien untereinander. Allerdings gerät Journalismus zunehmend unter den Einfluss von speziellen Interessengruppen. Er sieht sich zumindest teilweise durch einflussreiche Unternehmen und Verbände instrumentalisiert.

Zentral- und Osteuropa weisen spezifischere Mechanismen der Überwachung und Kontrolle auf. Für die Sowjetunion etwa gilt: „bad news are not news“ (S. 176). Die Katastrophe von Tschernobyl kann als ein Test der neuen Informationspolitik verstanden werden. Wesentlich funktionierte die Berichterstattung als eine Art Gegenpropaganda zur „provokativen“ westlichen Berichterstattung. Die alten Muster sind über lange Zeit hinweg noch immer stärker als die Versprechungen von Glasnost. Jedenfalls funktioniert die alte Hierarchie der Quellen Mitte der 80er Jahre in der Sowjetunion noch geradezu perfekt.

Amerikanische Erfahrungen artikuliert Hanno Hardt mit einem Artikel über „The Industrialization of News“. Journalismus entwi- ckelt sich von einem politischen Unternehmen zu einem kommerziellen Produkt für den Massenkonsum. Marketingstrategien beginnen, das Wesen der Nachricht grundlegend zu verändern. So ist es nicht verwunderlich, dass in Nachrichten zunehmend Verkaufsinformationen mit eingeschlossen werden. Aufgrund der Vermischung von Kapitalismus und Demokratie sieht der Autor eine industrialisierte Nachrichtenproduktion. Nachrichten werden nach Ansicht von Hardt standardisiert in Bezug auf Geschmackskulturen hergestellt. Nachrichten dringen zudem in die Privatheit der Bürger ein, sie trivialisieren die Politik. Mit anderen Worten: Journalismus engagiert sich als Showbusiness.

Kevin G. Barenhorst erörtert die „News Ideology in the $20^{\text {th }}$ Century”. Der Produktions- und Präsentationsprozess von Nachrichten ist fragmentiert und episodisch. Imperative des Marketings dominieren, auch bedingt durch eine zunehmend begrenzte Aufmerksamkeit des Publikums. Mit seiner ideologiekritischen Inhaltsanalyse zeigt der Autor, dass - entgegen langläufiger Annahmen - die Länge von Nachrichten in den letzten Jahrzehnten gewachsen ist. Außerdem werden in den Meldungen einzelne Handlungsträger (der Bürger) zunehmend durch Experten verdrängt; zugleich und stattdessen treten Journalisten selbst als Experten auf. Nachrichten berichten über weniger Ereignisse in längeren Meldungen; Ereignis- und Lokalbezug sinken. Barenhorst beschreibt somit die widersprüchlichen Bedingungen eines Nachrichtenjournalismus im Spannungsfeld von journalistischen Kriterien und ökonomischen Zwängen.

Im Epilog erklärt Horst Pöttker nochmals kulturelle und politisch-ökonomische Bedingungen als relevant für das Nachrichten-Paradigma. Historisch gesehen verbreitete sich das moderne Nachrichten-Paradigma ausgehend von den USA und England zunächst in Skandinavien und erst relativ spät, nämlich nach 1945 in Deutschland; in Osteuropa erst ab 1990. Ob diese Entwicklung allerdings mit dem viel verwendeten Begriff der Modernisierung zutreffend beschrieben ist, sei dahingestellt.

Insgesamt führt der Band zu facettenreichen medienhistorischen Einsichten und stellt sich als lohnenswertes Projekt dar. Ergänzend zu fragen ist allerdings, ob nicht einzelne Elemente des Nachrichten-Paradigmas zu einem früheren Zeitpunkt betrachtet werden 
könnten und müssten. Möglicherweise sind auch bestimmte Regionen, wie etwa in Mitteldeutschland der Raum Halle / Jena / Weimar zu berücksichtigen und relevant, da sich dort signifikante Startkulturen für das Nachrichtenparadigma auffinden lassen. Zudem gehen nicht alle Beiträge - wie angemerkt - systematisch und umfassend auf die fünf Elemente des Nachrichten-Paradigmas ein. Möglicherweise hätte das aber den Rahmen und die Möglichkeit einzelner Beiträge dieses Sammelbandes gesprengt. Zumal die historischen Entwicklungen in den angelsächsischen Ländern, in Deutschland sowie in Osteuropa zum Teil außerordentlich ungleichzeitig verlaufen.

Georg Ruhrmann

\section{Ulrich Saxer}

\section{Politik als Unterhaltung}

Zum Wandel politischer Öffentlichkeit in der Mediengesellschaft

Konstanz: UVK, 2007. - 345 S.

(Forschungsfeld Kommunikation; 20)

ISBN 978-3-89669-473-7

Dies ist mehr als ein Buch - es ist ein Werk. Statt nur erneut zu fordern, doch endlich einmal den Dingen auf den Grund zu gehen, legt Ulrich Saxer ein Denkmuster vor.

Auf Basis einer funktionalistischen Theorie sozialer Systeme entwickelt er ein umfassendes Modell politischer Öffentlichkeit, mit dem die grundlegenden Veränderungen der politischen Kommunikation erfasst und erklärt werden können. Ausgangspunkt ist ein zentrales Moment gesellschaftlicher Evolution - die Ausdifferenzierung des Mediensystems. Es löst sich aus der engen Verklammerung mit der Politik und orientiert sich stärker an einer ökonomischen Logik. Dadurch dynamisiert sich seine Entwicklung. Technische und organisatorische Innovationen ermöglichen ihm Leistungssteigerungen, die zu einer umfassenden und tiefgreifenden Durchdringung gesellschaftlicher Kommunikation führen - zur Medialisierung der Gesellschaft und damit zur Herausbildung einer Mediengesellschaft. Komplementär dazu wandelt sich das politische System. Seine Funktion, bindende Entscheidungen zu setzen und durchzusetzen, ist in mehrerer Hinsicht problematisch geworden. Auf der Mikroebene führt die Individualisierung zu einem
Mentalitätswandel: Schwindenden politischen Loyalitäten stehen wachsende und divergierende Erwartungen an die Fähigkeit der Politik gegenüber, Probleme rasch und reibungslos zu lösen. Auf der Mesoebene verstärkt sich die Konkurrenz gesellschaftlicher Interessen und ihrer politischer Organisationen um Einfluss auf die Entscheidungen. Und auf der Makroebene gewinnen die Überschneidungszonen der Politik mit anderen sozialen Systemen an Gewicht - wie mit dem Wirtschafts- oder dem Mediensystem. Die Systeme sind verstärkt auf die Leistungen der jeweils anderen angewiesen. Die konfliktgeladene Verknüpfung von politischem und Mediensystem in Form der Mediendemokratie lässt sich am Wandel der Öffentlichkeit ablesen. In demokratischen Staaten ist die Legitimität bindender Entscheidungen an eine möglichst weit gehende öffentliche Sichtbarkeit des Entscheidungsprozesses und an die Responsivität gegenüber der öffentlichen Meinung gebunden. Doch Form und Inhalt öffentlicher Kommunikation sind in einer Mediengesellschaft nicht mehr einfach politisch instrumentalisierbar oder dominierbar - da kann auch Medienpolitik nur noch begrenzt helfen. Öffentliche Aufmerksamkeit ist eine knappe Ressource, die fast alle brauchen und um die sich fast alle streiten. Dadurch wird Öffentlichkeit umkämpfter, komplexer und volatiler. Politik muss ihren Platz in der Öffentlichkeit gegen vieles andere durchsetzen und öffentliche Resonanz immer wieder neu erobern, auch dadurch, dass es sich den Bedingungen einer medial formatierten Öffentlichkeit unterwirft. Ein ebenso zentrales wie umstrittenes Moment öffentlicher Kommunikation ist Unterhaltung - einem individuellen und kollektiven "Spannungsmodulator“, der auch in politischer Kommunikation seinen Platz einnimmt. Und so versuchen politische Akteure, statt sich mit herkömmlichen Angeboten gegen Entertainment durchsetzen zu wollen, sich durch „Politainment" öffentliche Resonanz und Akzeptanz zu verschaffen, z. B. durch „Eventpolitik“ oder durch eine Emotionalisierung politischer Themen. Diese Kommunikationsstrategie wird von manchen Akteuren mehr angewandt als von anderen und ist in manchen Politikfeldern eingeführter als in anderen, aber sie prägt insgesamt politische Kommunikation. Dies löst politische Probleme und schafft zugleich neue auf der Mikro-, Meso- und Makroebene der politischen Kom- 